Backache

\title{
A rare cause of backache in an adult male
}

\section{Trikha, V Gupta}

Answers on $p 761$.

\begin{abstract}
A
45 year old man complained of pain in the lower back for one year. He presented with constipation and hesitation in passing urine for one month. The pain had been increasing in severity over the past three months. The patient had to take regular enemas for complete bowel evacuation. There was no history of weight loss, anorexia, or trauma. He had no weakness or radiation of pain to the lower limbs and had no symptoms of neurogenic claudication. The patient also noticed that he could not lie down straight on the lower back. On examination, there was diffuse swelling in midline over sacrum of size $4 \times 4$ $\mathrm{cm}$ with no local rise in temperature. $\mathrm{He}$ had sensory deficit in the perineal region. The knee and ankle jerks were normal with no motor weakness of the lower limbs. The haemogram and serum chemistry was within normal limits. Plain radiographs and magnetic resonance imaging (MRI) of the lumbosacral spine were performed (figs 1 and 2).
\end{abstract}

\section{QUESTIONS}

(1) Describe the radiographic and MRI findings.
(2) What is the most likely diagnosis?

(3) What is its management?

Postgrad Med J 2002;78:760

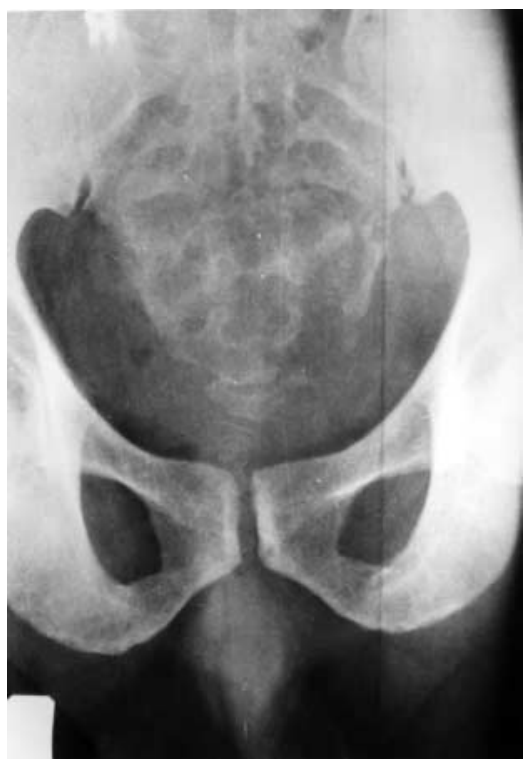

Figure 1 Anteroposterior radiograph of sacrum.
Authors' affiliations

V Trikha, V Gupta, Department of

Orthopaedics, All India Institute of Medical Sciences, New Delhi 110029 , India

Correspondence to: Dr Gupta; vikasgupta69@yahoo.com

Submitted 17 July 2002

Accepted 27 August 2002

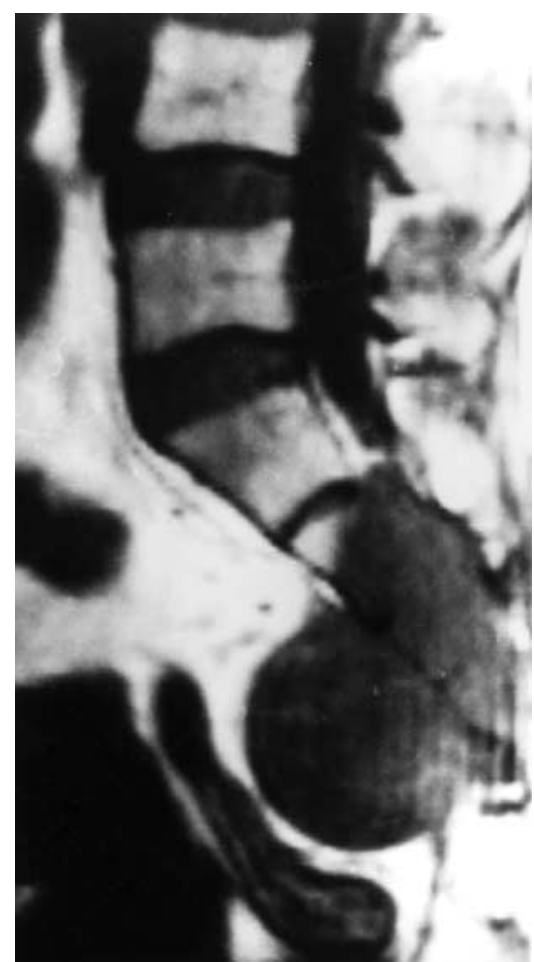

Figure $2 \mathrm{MRI}$ scan of sacrum

\begin{abstract}
A 70 year old man was found collapsed and acutely breathless in his toilet and was rushed to the local accident and emergency department. On arrival he was found to be dyspnoeic and unconscious with a Glasgow coma scale of 3. He had a temperature of $35.8^{\circ} \mathrm{C}$, pulse rate of 120 beats/min regular, and blood pressure 250/125 mm Hg. His respiratory rate was 28 breaths/min and oxygen saturations were $84 \%$ on a fractional inspiratory
\end{abstract}

oxygen of 0.60 . There were generalised widespread coarse crepitations in his chest and pink frothy sputum in his mouth. Both pupils were constricted and reacted poorly to light. He had generalised hypotonia, absent tendon reflexes, and unresponsive plantars. Accompanying relatives denied any past medical history or regular medication intake.

He was immediately intubated and ventilated by an anaesthetist. His arterial blood gases showed type I respiratory

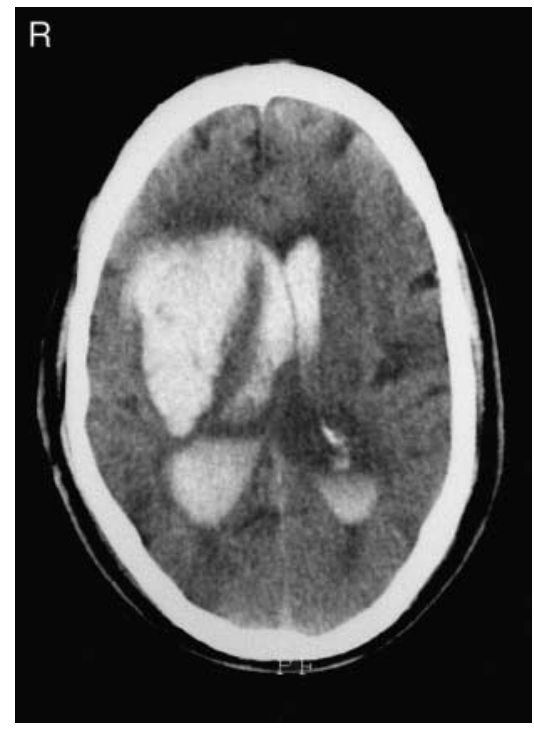

Figure 1 Computed tomogram of head. 
failure. His full blood count, urea and electrolytes, and glucose were normal. Serial electrocardiograms (ECGs)

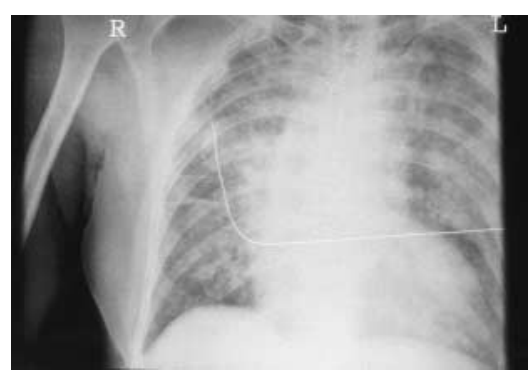

Figure 2 Chest radiograph at presentation. showed sinus tachycardia only and no signs of myocardial ischaemia. His creatine kinase and subsequent troponin-T were normal. He had emergency computed tomography of his head (fig l), which showed a large parenchymal bleed in the right cerebral hemisphere with extension into both lateral ventricles and the third and fourth ventricles and resultant hydrocephalus. He also had chest radiography (fig 2).

\section{QUESTIONS}

(1) What abnormalities are seen on the chest radiograph?

(2) What is the most likely diagnosis?
(3) What is the pathophysiology of this condition?

(4) How would you manage this patient? Postgrad Med J 2002;78:761

\section{Authors' affiliations}

N V Athavale, L H Soo, Department of Medicine, Rotherham General Hospital, Rotherham

Correspondence to: $\operatorname{Dr} \mathrm{N} V$ Athavale, Department of Medicine for the Elderly, Rotherham General Hospital, Moorgate Road Rotherham S60 2UD, UK; nathavale49@hotmail.com

Submitted 1 July 2002

Accepted 4 September 2002

\section{SELF ASSESSMENT ANSWERS}

\section{A rare cause of backache in an adult male}

\section{Q1: Describe the radiographic and MRI findings}

The anteroposterior radiograph of lumbosacral spine reveals a lytic lesion of the sacrum involving the S2-4 vertebra. MRI reveals a destructive mass lesion involving the S2-4 vertebra. The mass is involving the sacral canal displacing the sacral nerve roots at these levels. Associated pelvic component of the mass is predominantly seen at the S4 level. The rectum and the bladder appear to be free in the MRI scan.

\section{Q2: What is the most likely diagnosis?} The most likely diagnosis is sacral chordoma. The MRI findings plus the age of the patient and the classical findings of low back pain with constipation, urinary hesitancy, and sacral mass makes sacral chordoma the diagnosis

\section{Q3: What is its management?}

The primary curative treatment of sacral chordoma is surgery. Total surgical extirpation offers the best chance for cure. When radical excision cannot be achieved, adjuvant therapy plays an important part. Radiotherapy has been used in chordomas for palliation and for radical treatment in patients with residual disease after partial resection.

\section{Discussion}

Chordoma is a rare tumour arising from the primitive notochord located along the axia skeleton. ${ }^{1}$ More than half of these tumours arise in the sacral region, $30 \%-40 \%$ from the clivus, and the rest (about 15\%) arise elsewhere in the axial skeleton. ${ }^{2}$ Chordoma is the most frequent primary malignant tumour of the sacrococcygeal region. The average age of

Box 1: Clinical presentation of sacral chordoma

- Low backache.

- Urinary retention.

- Incomplete bowel evacuation.

- Radicular leg pain.

- Sacral mass. onset is in late adulthood with males being affected two to three times more frequently than females. Due to their insidious onset and vague clinical symptoms, the time lapse between the onset of symptoms and diagnosis is more than a year. Since the visceral and vital neurological structures lie in vicinity, the ideal treatment of wide excision is difficult. Sacral chordomas are slow growing, locally aggressive tumours with a high recurrence rates.

The earliest and the most common symptom of the sacral chordomas is sacral pain (box 1). Initially, the pain may be discontinuous and not well localised. With the invasion of sacrum and the nerve roots, it becomes intractable and localised to the anal region. The nerve root compression suggests tumour invasion into the foramina producing symptoms such as urinary complaints, constipation, and radicular leg pain. These complaints may lead to a false diagnosis of haemorrhoids, pilonidal sinus, or prolapsed intervertebral disc. ${ }^{4}$ Urine or faecal incontinence, intestinal obstruction, bleeding from the rectum and bladder are seen when the disease has spread extensively.

On physical examination, the tumour is usually felt between the rectum and sacrum. At rectoscopy, the rectal mucosa is found to be normal and intact. In general, sacral chordomas may spread to regional lymph nodes but visceral organ metastasis is rarely seen. Bone destruction is the most prominent finding in sacral chordomas. Radiologically, they may be confused with chondroma, chondrosarcomas, and giant cell tumour. ${ }^{4}$ All other presacral tumours should also be included in the differential diagnosis (box 2).

As a rule, chordoma should be diagnosed histologically. A biopsy or fine needle aspiration cytology (FNAC) should be performed only via a posterior route. A transrectal biopsy should not be done as it may lead to rectal seeding of the tumour. ${ }^{6}$ Microscopically, it consists of pleomorphic cells arranged in clusters amid myxoid matrix. Spindle shaped cells are also seen. These cells show vacuolated cytoplasm with prominent vesicular nuclei.

The modern imaging techniques of computed tomography and MRI have helped in the earlier diagnosis and better delineation of the extent of the tumour. A chordoma contrasts highly with the adjacent soft tissue in T2 weighted images. MRI helps in better surgical planning, leading to less radical surgery, decreased morbidity, and better long term outcome. It is also useful for evaluation of recurrence in follow up.

A radical excision is the treatment of choice for sacral chordomas. The average survival from the onset of symptoms for sacral chordomas is usually 6.4 years. ${ }^{7}$ Bladder and rectal functions after surgery are major problems. If bilateral sacral nerve roots are sectioned, normal urogenital and rectal functions are lost. However, it is imperative that surgical margins should not be compromised, as the next surgery after local relapse becomes very difficult and will involve the resection of many more nerve roots. Since radical surgery is feasible only in a few cases, radiotherapy plays an important part. It has been attempted in cases for palliation and for radical treatment in patients with residual disease after subtotal or partial resection. Postoperative irradiation for local recurrence helps improve the quality of life, as it effectively relieves pain. ${ }^{8}$ Recent advances in radiation therapy like particle therapy (proton beam) offer great potential for treating residual disease with minimum normal tissue toxicity.

The prognosis of patients with sacral chordoma is reported to be dismal and attributable to the usual intralesional surgery performed (box 3). It is imperative that even the biopsy or FNAC be performed in referral tumour centres to reduce the risk of local recurrence, metastasis, and decreased survival. ${ }^{9}$

In conclusion, effective management of sacral chordoma involves early diagnosis,

Box 2: Differential diagnosis of sacral chordoma.

- Chondroma

- Chondrosarcoma.

- Giant cell tumour.

- Fibrosarcoma.

- Tuberculosis.

- Hydatid cyst.

- Aneurysmal bone cyst.

- Neurofibroma.

- Schwannoma.

- Sacrococcygeal teratoma.

- Protruded intervertebral disc.

- Lymphoma

- Metastatic tumour.

- Rectal carcinoma. 
Box 3: Adverse prognostic factors

Local recurrence

- Invasive diagnostic procedure outside tertiary fumour centre.

- Larger tumour size.

- Inadequate surgical margins.

- Presence of microscopic tumour necrosis.

Metastasis

- Invasive diagnostic procedure outside tumour centre.

- Ki 67 positivity in $>5 \%$ of the tumour cells.

Tumour related death

- Inadequate surgical margins at definitive surgery.

- Larger tumour size.

adequate surgical resection with tumour-free margins, followed by high dose radiotherapy and close follow up.

\section{Final diagnosis}

Sacral chordoma.

\section{References}

1 Heffelfinger MJ, Dahlin DC, MacCarty CS, et al. Chordomas and cartilaginous tumours at the skull base. Cancer 1973:32:410-20.

2 Sundaresan N, Galicich JH, Chu FCH, et al. Spinal chordomas. J Neurosurg 1979;50:312-19

3 Chetty R, Levin CV, Kalan MR. Chordoma. A 20 year clinicopathologic review of the experience of Groote Schur Hospital, Cape Town. J Surg Oncol 1991:46:261-4.

4 Turgut M, Gul B, Taskin Y. Sacrococcygeal chordomas: problems in diagnosis and management. Arch Orthop Trauma Surg 1998;117:100-2.

5 Beagie JM, Mann CV, Butler CB. Sacrococcygeal chordoma. Br J Surg 1969:56:586.

6 Bethke KP, Neifield JP, Lawrence W Jr. Diagnosis and management of sacrococcygeal chordomas. J Surg Oncol 1991:48:232-8.

7 McMaster ML, Goldstein AM, Bromley CM, et al. Chordoma: incidence and survival patterns in the United States, 1973-1995 Cancer Causes and Control 2001;12:1-11.

8 Cummings BJ, Hodson DI, Bush RS. Chordoma: the results of megavoltage radiation therapy. Int J Radiat Oncol Biol Phys 1983:9:633-42

9 Bergh P, Kindblom LG, Gunterberg B, et al. Prognostic factors in chordoma of the sacrum and mobile spine. A study of 39 patients. Cancer 2000;88:2122-34.

\section{A drowsy breathless man}

Q1: What abnormalities are seen on the chest radiograph?

There is diffuse bilateral alveolar shadowing with perihilar haziness in keeping with clinical features of pulmonary oedema.

\section{Q2: What is the most likely diagnosis?}

In the absence of any evidence of cardiac event and considering his computed tomography findings this man could have neurogenic pulmonary oedema.

\section{Q3: What is the pathophysiology of} this condition?

The exact pathophysiology of neurogenic pulmonary oedema is not clearly understood. It is thought to be due to a combination of raised pulmonary capillary pressure and increased capillary permeability.

\section{Q4: How would you manage this patient?}

The principles of treatment are reduction of intracranial pressure, diuresis, and reversing the effects of excess sympathetic activity.

\section{Discussion}

In our patient, who presented with acute pulmonary oedema, it was important to rule out a cardiac cause first, even in the absence of any significant past medical history. His ECGs and normal cardiac markers excluded a possibility of ischaemic cardiac event. As he was unconscious at presentation without any signs of type 2 respiratory failure or metabolic abnormalities on his blood tests, he underwent computed tomography of the head, which revealed a large intracerebral bleed (fig $\mathrm{l}$; see $\mathrm{p} \mathrm{760)}$ ). This raises the likelihood of neurogenic pulmonary oedema in the absence of a cardiac cause for pulmonary oedema in a patient who is unconscious with a primary intracranial event and is acutely breathless.

Neurogenic pulmonary oedema is a rare but dangerous complication of intracranial injury. It has been reported after subarachnoid haemorrhage, head injury, grand mal seizures, and intracranial bleeding. ${ }^{1-3}$ In the majority of cases features of pulmonary oedema may be evident within a few minutes of the cerebral insult. The incidence is hard to determine as less severe cases may be unrecognised or attributed to aspiration. It is estimated to occur, however, in fewer than $10 \%$ of patients with subarachnoid haemorrhage. ${ }^{1}$ It is related to the severity of the cerebral injury and is rarely seen in patients with normal level of consciousness. The typical clinical picture consists of unexpected dyspnoea, cyanosis, and production of pink sputum. Patients are pale, sweaty, and hypertensive and semiconscious. Absence of evidence for cardiac event is a clue.

The pathophysiology of neurogenic pulmonary oedema is not fully understood. An acute cerebral insult can lead to massive sympathetic discharge mediated by anterior hypothalamus. This leads to a large increase in systemic and pulmonary blood pressures while cardiac output falls due to increased peripheral resistance. ${ }^{2}$ Hence there is redistribution of blood volume from systemic to pulmonary circulation. This leads to pulmonary venous and left atrial engorgement. A subsequent rise in pulmonary capillary pressures also damage endothelial cells leading to increased pulmonary capillary permeability. ${ }^{14}$

Treatment is aimed at reduction of intracranial pressure, diuresis, and reversing peripheral effects of sympathetic stimulation ${ }^{5}$ Controlled hyperventilation with intermittent positive pressure ventilation and surgical decompression are used to reduce intracranial pressure. Diuretics are used to control blood volume and intracranial pressure. It has been claimed that labetolol and chlorpromazine are also beneficial. ${ }^{1}$ The depression of myocardial function is shown to be reversed by dobutamine and a vasodilator. ${ }^{26}$ Patients are treated in intensive care unit till they are stabilised. Mortality is very high and survivors $(<10 \%)$ would be left with significant disability and would need prolonged rehabilitation in appropriate cases.

The patient reported here survived the initial events including episodes of sepsis on intensive care unit. He was left with significant disability as he was aphasic, bed bound, and fed by gastrostomy. At three monthly review he had no clinical signs of heart failure

\section{Final diagnosis}

Neurogenic pulmonary oedema secondary to intracerebral haemorrhage.

\section{References}

1 Warlow CP, Dennis MS, van Gijn J, et al. Specific treatment of aneurismal subarachnoid haemorrhage. Stroke-a practical guide to management. London: Blackwell Science, 2001: 546-7

2 Kerr G. Neurogenic pulmonary oedema. J Accid Emerg Med 1998;15:275-7.

3 Brambrink AM, Tzanova I. Neurogenic pulmonary oedema after a generalised epileptic seizure. Eur J Emerg Med 998;5:59-66

4 Theodre J, Robin ED. Pathogenesis of neurogenic pulmonary oedema. Lance 1975;ii:749-51.

5 Wauchob TD, Brooks RJ, Harrison KM Neurogenic pulmonary oedema. Anaesthesia 1984:39:529-34

6 Parr MJ, Finfer SR, Morgan MK. Reversible cardiogenic shock complicating subarachnoid haemorrhage. BM 1996:313:681-3. 